\title{
Depressão e suas implicações no aleitamento materno
}

\author{
Depression and its implications in breast feeding
}

\section{Márcia Regina Vitolo ${ }^{1}$, Silvia Pereira da Cruz Benetti ${ }^{2}$, Gisele Ane Bortolini' ${ }^{1}$, Angelice Graeff ${ }^{2}$, Maria de Lourdes Drachler ${ }^{3}$}

${ }^{1}$ Programa de Pós-Graduação em Ciências Médicas, Fundação Faculdade Federal de Ciências Médicas de Porto Alegre (FFFCMPA), Porto Alegre, RS. ${ }^{2}$ Universidade do Vale do Rio dos Sinos (UNISINOS), São Leopoldo, RS. ${ }^{3}$ School of Allied Health Professions, University of East Anglia, Norwich, Inglaterra.

\section{Resumo}

Objetivo: O objetivo deste estudo foi avaliar a prevalência de sintomas depressivos num grupo de mães de crianças entre 12 e 16 meses do município de São Leopoldo (RS) e suas associações com tempo de aleitamento materno e aspectos do desenvolvimento da criança.

Método: Foram avaliadas 263 mães de crianças recrutadas ao nascimento em uma maternidade que atende população de baixo nível socioeconômico. Os sintomas depressivos foram avaliados por meio do Inventário para Depressão de Beck.

Resultados: Foi observado que 35,7\% das mães apresentaram sintomas de depressão. Quanto à classificação, 18,3\% apresentaram depressão leve, $11 \%$, depressão moderada, e 6,5\%, grave. As mães sem companheiros (razão de prevalência $-\mathrm{RP}=1,70$; IC95\% = $1,20-2,38$ ) e provenientes de famílias não-nucleares apresentaram mais sintomas depressivos (RP = 1,38; IC95\% = 0,99-1,92). As freqüências de aleitamento materno exclusivo por 6 meses $(\mathrm{RP}=1,86$; IC95\% = 0,94-3,68) e aleitamento materno aos 12 meses (RP $=1,80$; IC95\% = 1,26-2,58) foram maiores no grupo de mães sem sintomas depressivos.

Conclusão: É necessária maior atenção à saúde mental das mulheres, considerando a alta prevalência de sintomas depressivos nessa população e a forte associação com o menor tempo de aleitamento materno.

Descritores: Sintomas depressivos, mães, criança, aleitamento materno, desenvolvimento.

\begin{abstract}
Objective: This study aimed at verifying the incidence of depressive symptoms in a group of mothers of children between 12 and 16 months in the municipality of São Leopoldo (RS, Brazil) and their associations with breast feeding and overall child development. Method: This study included 263 mothers who delivered at a hospital providing services to a population of low socioeconomic level. Depressive symptoms were assessed using Beck Depression Inventory.

Results: It was observed that $35.7 \%$ of mothers presented depressive symptoms. As to classification, $18.3 \%$ had mild, $11 \%$ moderate and 6.5\% severe depression. Mothers without partners (prevalence ratio $-\mathrm{PR}=1.70$; IC95\% $=1.20-2.38$ ) and mothers from nonnuclear families presented more depressive symptoms $(\mathrm{PR}=1.38$; IC95\% $=0.99-1.92)$. Exclusive breast feeding at $6(\mathrm{PR}=1.86$; IC95\% $=0.94-3.68)$ and 12 months (PR = 1.80; IC95\% = 1.26-2.58) was more frequent in the group of mothers without depressive symptoms.

Conclusion: More attention should be given to women's mental health, considering the high prevalence of depressive symptoms in this population and the strong association with less breastfeeding time.

Keywords: Depressive symptoms, mothers, children, breast feeding, development.
\end{abstract}

Correspondência:

Silvia Pereira da Cruz Benetti, Rua Riveira, 150/301, CEP 90670-160, Porto Alegre, RS. E-mail: sbenetti@unisinos.br

Copyright (C) Revista de Psiquiatria do Rio Grande do Sul - SPRS

Recebido em 19/04/2007. Aceito em 06/05/2007. 


\section{Introdução}

Diversos estudos sobre o estado afetivo materno no primeiro ano de vida do bebê indicam que a ocorrência de quadros depressivos está associada a alterações no desenvolvimento infantil das áreas neurológica, cognitiva e emocional ${ }^{1-5}$, com efeitos duradouros ao longo do desenvolvimento da criança, que podem perdurar até a adolescência ${ }^{6}$. Fundamentalmente, os quadros depressivos interferem em diversos aspectos do funcionamento e estado afetivo materno e, conseqüentemente, afetam a qualidade da relação entre a mãe e seu bebê.

No âmbito do comportamento e da capacidade de sensibilidade materna, observam-se dificuldades nas trocas afetivas, estabelecimento de apego inseguro $^{5,7}$ e incapacidade materna de sensibilidade ao choro do bebê ${ }^{8}$. Mães depressivas também apresentam maiores dificuldades em engajarem-se em cuidados de saúde, tanto pessoal como do bebê maior prevalência de uso de tabaco, não-aderência a cuidados de saúde do bebê e negligência ${ }^{9}-$, amamentam com menor freqüência seus bebês, interrompem a amamentação no peito mais cedo e percebem-se menos confiantes quanto à sua capacidade de amamentar ${ }^{10}$.

Em geral, a maioria dos estudos identifica prevalência de 10 a $15 \%$ de casos de depressão materna no primeiro ano de vida da criança ${ }^{4,11-13}$. Inúmeras vezes, os casos de depressão materna não são identificados pelos profissionais de saúde ${ }^{2}$, situação que intensifica a gravidade do problema. Dessa maneira, são necessários estudos populacionais que investiguem índices de prevalência e fatores de desenvolvimento associados à depressão para a obtenção de dados e de indicadores que permitam o incremento da deteç̧ão precoce e do conhecimento dos profissionais de saúde sobre o problema. Portanto, este estudo teve como objetivo verificar a prevalência de sintomas depressivos num grupo de mães de crianças com idade de 12 a 16 meses de vida do município de São Leopoldo e as possíveis associações com o tempo de aleitamento materno, desenvolvimento geral da criança, condições ambientais e relacionais entre mãe/filho(a) e características sociodemográficas, como educação, estado civil, situação de emprego e idade.

\section{Método}

Este estudo faz parte de uma investigação maior, realizada na região sul do Brasil, sobre o impacto da implementação de um programa de orientação nutricional - “Dez Passos da Alimentação Saudável”14
-, em uma coorte de crianças durante o primeiro ano de vida, sobre as práticas alimentares, as condições de saúde e de desenvolvimento. Esse estudo e a metodologia detalhada foram publicados anteriormente ${ }^{15}$. Também foram avaliados dados sociodemográficos das famílias participantes. Definiu-se como família nuclear aquela composta por pai, mãe e filhos. Quando havia um ou mais membros familiares presentes em mesmo núcleo familiar, categorizou-se como não-nuclear. As crianças que participaram do estudo nasceram no hospital público municipal. Foram identificados os recém-nascidos que tivessem peso de nascimento maior de 2.500 g e idade gestacional maior que 37 semanas. Os critérios de exclusão foram: mães HIV-positivas, má-formação congênita e parto múltiplo. Após a identificação dos recém-nascidos que preenchiam os requisitos, as mães foram esclarecidas com relação ao estudo e consultadas quanto à aceitação para participar.

O cálculo do tamanho da amostra do primeiro projeto baseou-se em uma freqüência de aleitamento materno exclusivo (AME) até os 4 meses de 21,6\% no grupo-controle e estimou uma diferença de $65 \%$ na freqüência dessa prática entre os grupos, após a intervenção. Outros parâmetros para esse cálculo foram: poder de $80 \%$ e nível de confiança de $95 \%$, o que determinou um tamanho amostral de 177 crianças em cada grupo, totalizando 354 crianças. Considerando uma previsão de perdas de $25 \%$, foram recrutados 500 pares mãe/filho ao nascimento para que o número amostral fosse atingido. No final do primeiro ano de vida, 397 crianças completaram o estudo. Os dados detalhados das perdas foram publicados anteriormente ${ }^{15}$. Entretanto, a avaliação da depressão ocorreu após 129 crianças já terem sido avaliadas. A amostra de 263 mães que participaram da investigação sobre depressão teve poder estatístico para testar a hipótese de que a depressão interfere na amamentação, considerando a prevalência de 51,1\% de crianças em aleitamento materno nas mães que apresentaram sintomas depressão e 69,6\% de crianças em aleitamento materno nas mães que não apresentaram sintomas de depressão, ao final do primeiro ano. O número amostral necessário seria de 238 crianças, com poder de $80 \%$ e nível de confiança de $95 \%$. As visitas ocorreram de outubro de 2001 a junho de 2002 e foram realizadas por estudantes de nutrição treinados pelos pesquisadores da área de psicologia e nutrição.

\section{Depressão materna}

Para a avaliação da presença e gravidade da depressão materna, foi utilizado o Inventário para Depressão de Beck, um instrumento de fácil aplicação 
que tem como propriedade avaliar manifestações comportamentais de depressão na semana anterior à aplicação ${ }^{16}$. Neste trabalho, utilizamos o ponto de corte maior ou igual a 12 para a categorização de sintomas depressivos.

\section{Avaliação do desenvolvimento}

Foi utilizado o Teste de Triagem de Desenvolvimento de Denver (Denver II Developmental Screening Test - DDST-R $)^{17}$, o qual abrange a avaliação do desenvolvimento motor-grosseiro, motor-adaptativo, pessoal-social e de linguagem, de zero a 6 anos de idade. Considerou-se suspeita de atraso de desenvolvimento motor amplo quando a criança não era capaz de desempenhar corretamente um aspecto que $75 \%$ das crianças da população de referência, com idade inferior, conseguiram. Essas estimativas se basearam na padronização dos aspectos desse teste para crianças brasileiras realizada em um estudo do desenvolvimento de crianças de zero a 5 anos de idade em Porto Alegre ${ }^{18}$. A escolha dos aspectos correspondeu a todas as atividades realizadas por pelo menos $75 \%$ das crianças na faixa etária dos 12 meses.

\section{Home Observation for the Measurement of the Environment (HOME)}

O inventário HOME é um instrumento concebido para medir a qualidade e a quantidade de apoio social, emocional e cognitivo disponível para a criança em seu ambiente familiar ${ }^{19,20}$. No presente estudo, foram utilizadas as medidas de estimulação e envolvimento materno. Utilizando-se de um critério de corte baseado na freqüência de comportamentos positivos maternos em relação à interação e envolvimento com a criança e também no número de itens avaliados (11 itens), foram consideradas duas categorias de classificação: insatisfatório - mães com cinco ou menos $(\leq 5)$ comportamentos positivos de um total de 11 ; satisfatório - mães com mais de cinco (> 5) comportamentos positivos.

\section{Estrutura familiar}

As famílias que eram constituídas de pai, mãe, filho e/ou irmãos foram denominadas de família nuclear. As famílias que continham outras pessoas morando na mesma casa, como avós, tias e outros parentes ou amigos da família, foram denominadas de não-nuclear. Aquelas com ausência de pai ou mãe também foram denominadas de não-nuclear.

\section{Aleitamento materno}

Os dados sobre aleitamento materno foram obtidos com os seguintes critérios: AME - leite materno sem água, chá ou outro alimento qualquer; e aleitamento materno presença do leite materno independente de outros alimentos.

\section{Aspectos éticos}

O projeto de pesquisa "Implementação e Avaliação do Impacto do Programa de Promoção para a Alimentação Saudável” para crianças menores de 2 anos foi aprovado pelo Comitê de Ética de Pesquisa da Universidade Federal do Rio Grande do Sul.

\section{Análise estatística}

Todos os dados quantitativos foram inseridos no programa Epi-Info, versão 6.04, com dupla entrada de dados para validação. Os dados foram organizados de acordo com as análises no programa SPSS, e a partir das correções necessárias foram realizadas as análises, observando-se os resultados pelo teste qui-quadrado de Pearson e teste exato de Fischer (identificado na tabela) e as razões de prevalência (RP) para verificar quais as variáveis estavam associadas aos sintomas depressivos maternos. Utilizou-se intervalo de confianças 95\% (IC95\%), considerando-se 5\% o nível de rejeição das hipóteses de nulidade ( $<<0,05)$.

\section{Resultados}

Das 268 mães elegíveis para participar do estudo, cinco não estavam em casa no momento da entrevista, estando a criança sob cuidados de outro responsável. Do total de mães, 94 (35,7\%) apresentavam sintomas depressivos; 48 foram classificadas com níveis leve (18,3\%), 29, com moderado (11\%), e 17 , com grave $(6,5 \%)$.

O nível de renda familiar total, expresso por salários mínimos, mostrou que 2/3 das famílias viviam com três ou menos salários mínimos. A escolaridade materna foi menor que 8 anos de estudo em 50\% dos casos. Em relação à estrutura familiar, houve maior freqüência de casos com sintomas de depressão nas categorias de mães sem companheiros $(p=0,007)$ e provenientes de famílias não-nucleares $(\mathrm{p}=0,068)$ (Tabela 1$)$. 
Tabela 1 - Razões de prevalência da depressão materna de acordo com fatores sociodemográficos e práticas de aleitamento

\begin{tabular}{|c|c|c|c|c|c|c|}
\hline & \multicolumn{4}{|c|}{ Presença de sintomas depressivos } & \multirow{3}{*}{ RP (IC 95\%) } & \multirow{3}{*}{$\mathbf{p}$} \\
\hline & \multicolumn{2}{|c|}{ Sim } & \multicolumn{2}{|c|}{ Não } & & \\
\hline & $\mathbf{n}$ & $(\%)$ & $\mathbf{n}$ & $(\%)$ & & \\
\hline \multicolumn{7}{|l|}{ Salários mínimos } \\
\hline$\leq 3$ & 58 & $(65,9)$ & 117 & $(69,6)$ & 1 & \\
\hline$>3$ & 30 & $(34,1)$ & 51 & $(30,4)$ & $1,06(0,87-1,29)$ & 0,543 \\
\hline \multicolumn{7}{|l|}{ Idade Materna } \\
\hline$<20$ anos & 25 & $(26,6)$ & 30 & $(17,8)$ & 1 & \\
\hline$\geq 20$ anos & 69 & $(73,4)$ & 139 & $(82,2)$ & $0,82(0,63-1,06)$ & 0,091 \\
\hline \multicolumn{7}{|l|}{ Escolaridade materna } \\
\hline$<8$ anos & 54 & $(57,4)$ & 85 & $(50,3)$ & 1 & \\
\hline$\geq 8$ anos & 40 & $(42,6)$ & 84 & $(49,7)$ & $1,20(0,87-1,67)$ & 0,266 \\
\hline \multicolumn{7}{|l|}{ Ocupação da mãe } \\
\hline Não remunerada & 62 & $(66,7)$ & 111 & $(66,1)$ & 1 & \\
\hline Remunerada & 31 & $(33,3)$ & 57 & $(33,9)$ & $1,01(0,72-1,44)$ & 0,922 \\
\hline \multicolumn{7}{|l|}{ Estado civil } \\
\hline Sem companheiro & 21 & $(22,3)$ & 17 & $(10,1)$ & 1 & \\
\hline Com companheiro & 73 & $(77,7)$ & 152 & $(89,9)$ & $1,70(1,20-2,38)$ & 0,007 \\
\hline \multicolumn{7}{|l|}{ Estrutura familiar } \\
\hline Não nuclear & 31 & $(33,0)$ & 38 & $(22,6)$ & 1 & \\
\hline Nuclear & 63 & $(67,0)$ & 130 & $(77,4)$ & $1,38(0,99-1,92)$ & 0,068 \\
\hline \multicolumn{7}{|c|}{ Aleitamento materno exclusivo } \\
\hline$<4$ meses & 64 & $(68,1)$ & 98 & $(58,7)$ & 1 & \\
\hline$\geq 4$ meses & 30 & $(31,9)$ & 69 & $(41,3)$ & $1,30(0,91-1,86)$ & 0,134 \\
\hline$<6$ meses & 87 & $(92,6)$ & 140 & $(83,8)$ & 1 & \\
\hline Aos 6 meses & 7 & $(7,4)$ & 27 & $(16,2)$ & $1,86(0,94-3,68)$ & 0,045 \\
\hline \multicolumn{7}{|l|}{ Aleitamento materno } \\
\hline$<6$ meses & 46 & $(47,4)$ & 51 & $(30,4)$ & 1 & \\
\hline Aos 6 meses & 48 & $(51,1)$ & 117 & $(69,6)$ & $1,63(1,19-2,24)$ & 0,003 \\
\hline$<12$ meses & 64 & $(68,1)$ & 78 & $(46,4)$ & 1 & \\
\hline Aos 12 meses & 30 & $(31,9)$ & 90 & $(53,6)$ & $1,80(1,26-2,58)$ & 0,001 \\
\hline
\end{tabular}

$\mathrm{RP}=$ razão de prevalência.

A avaliação do período de AME mostrou freqüências semelhantes entre os grupos de mães com sintomas depressivos e o grupo de mães sem sintomas depressivos no período menor que 4 meses $(p=0,134)$. Entretanto, a freqüência de AME foi significativamente maior para o período de 6 meses no grupo de mães sem presença de sintomas depressivos, quando comparado com o grupo com sintomas de depressão $(p=0,045)$. Em relação à presença de aleitamento materno na última visita domiciliar, observou-se menor freqüência de mães que amamentavam seus filhos aos 12 meses de idade no grupo com sintomas depressivos, quando comparado com o grupo sem os sintomas $(\mathrm{p}=$ 0,001).

Considerando os comportamentos infantis aos 12 meses de idade, não foram encontradas diferenças significativas entre os grupos de mães com e sem depressão no desenvolvimento psicossocial, de linguagem e motor amplo das crianças. Em termos de estimulação do ambiente, ambos os grupos evidenciaram comportamentos similares em relação à criança, não havendo diferenças entre as mães com sintomas depressivos e as mães sem a presença de sintomas (Tabela 2). 
Tabela 2 - Razões de prevalência de depressão materna de acordo com os escores de desenvolvimento infantil

\begin{tabular}{|c|c|c|c|c|c|c|}
\hline & \multicolumn{4}{|c|}{ Presença de sintomas depressivos } & \multirow{3}{*}{ RP (IC95\%) } & \multirow{3}{*}{$\mathbf{p}$} \\
\hline & \multicolumn{2}{|c|}{ Sim } & \multicolumn{2}{|c|}{ Não } & & \\
\hline & $\mathbf{n}$ & $(\%)$ & n & $(\%)$ & & \\
\hline \multicolumn{7}{|l|}{ Desenvolvimento psicossocial } \\
\hline Jogar bola & 79 & $(84,1)$ & 129 & $(76,8)$ & $0,73(0,46-1,16)$ & 0,164 \\
\hline Dar tchau* & 90 & $(95,7)$ & 166 & $(98,8)$ & $1,89(1,05-3,42)$ & 0,192 \\
\hline Indicar desejos & 76 & $(80,8)$ & 144 & $(85,7)$ & $1,24(0,84-1,84)$ & 0,303 \\
\hline Brincar de esconder & 80 & $(85,1)$ & 144 & $(85,7)$ & $1,03(0,66-1,62)$ & 0,893 \\
\hline \multicolumn{7}{|l|}{ Desenvolvimento da linguagem } \\
\hline Uma palavra específica & 85 & $(90,4)$ & 149 & $(88,7)$ & $0,88(0,50-1,56)$ & 0,663 \\
\hline Duas palavras específicas & 67 & $(71,3)$ & 114 & $(67,9)$ & $0,90(0,63-1,29)$ & 0,566 \\
\hline \multicolumn{7}{|l|}{ Desenvolvimento infantil motor amplo } \\
\hline Caminha segurando mobília & 86 & $(91,5)$ & 158 & $(94,0)$ & $0,88(0,73-2,17)$ & 0,432 \\
\hline Abaixa-se e levanta-se sozinho & 46 & $(48,9)$ & 90 & $(53,9)$ & $1,13(0,82-1,57)$ & 0,442 \\
\hline \multicolumn{7}{|l|}{ Estimulação do ambiente } \\
\hline Pais brincam ou pegam no colo* & 91 & $(97,8)$ & 167 & $(99,4)$ & $1,89(0,83-4,28)$ & 0,290 \\
\hline Brinca com areia, água, terra ou comida & 62 & $(65,9)$ & 112 & $(66,7)$ & $1,02(0,73-1,43)$ & 0,907 \\
\hline Sai junto para as compras & 88 & $(93,6)$ & 150 & $(89,3)$ & $0,68(0,33-1,38)$ & 0,244 \\
\hline $\begin{array}{l}\text { Leva para as consultas médicas, mesmo } \\
\text { não estando doente }\end{array}$ & 51 & $(54,3)$ & 104 & $(61,9)$ & $1,22(0,88-1,69)$ & 0,227 \\
\hline Sai de casa pelo menos $4 \mathrm{x}$ por semana & 78 & $(82,9)$ & 144 & $(85,7)$ & $1,14(0,75-1,74)$ & 0,555 \\
\hline \multicolumn{7}{|l|}{ Interação mãe-criança } \\
\hline Insatisfatória $<5$ & 9 & $(10,1)$ & 5 & $(3,1)$ & 1 & \\
\hline Satisfatória $\geq 5$ & 80 & $(89,9)$ & 158 & $(96,9)$ & $1,91(1,24-2,94)$ & 0,020 \\
\hline
\end{tabular}

* Teste exato de Fischer

$\mathrm{RP}=$ razão de prevalência.

O envolvimento materno, entretanto, foi diferente no grupo de mães com sintomas depressivos, sendo observado maior número de mães classificadas como “insatisfatório” em relação a comportamentos positivos observados na interação mãe/criança (Tabela 2).

\section{Discussão}

A identificação de sintomas depressivos maternos na gestação e no primeiro ano de vida do bebê é de fundamental importância para a promoção de ações preventivas dirigidas à saúde materno-infantil. Extensa revisão dos principais estudos sobre depressão materna e a relação mãe/bebê apontam que mesmo as situações menores de depressão podem ser potencialmente prejudiciais à saúde do bebê $\hat{e}^{1,7}$.

Estudos realizados em países desenvolvidos mencionam índices de depressão materna de 10 a 15\% no primeiro ano de vida da criança ${ }^{2,11}$. Outro estudo encontrou índice de 12\% num grupo de 434 mães que estavam com bebês com 12 meses de idade ${ }^{12}$. Entretanto, na África do Sul, foram encontrados valores maiores numa investigação sobre a prevalência de depressão puerperal e distúrbios na relação mãe/filho em 147 mulheres com bebês de 2 meses de idade. O índice de depressão maior foi de $34,7 \%$ entre o grupo de mães investigadas. Os autores consideram que esse valor de três vezes mais casos de depressão do que amostras de países desenvolvidos segue a mesma tendência de outros estudos com populações em situação de risco econômico e social, apontando para a importância de investigações nesses grupos ${ }^{2}$. Da mesma forma, em investigação realizada na Índia, identificou-se que 23\% de mulheres, em torno do segundo mês de gravidez, apresentaram sintomas depressivos e que metade delas ainda mantinha os sintomas 6 meses depois, sugerindo que a depressão materna fosse categorizada como um problema de saúde pública $^{21}$. Outro estudo também menciona índices em torno de 30\% no sul da Ásia ${ }^{22}$.

No Brasil, os índices de depressão materna refletem igualmente os dados mencionados nos estudos 
internacionais. Um estudo realizado com grupo de 77 mulheres da cidade de São Paulo, após um período que variava entre 3 e 6 meses depois do parto, identificou índice de quase 33\% de mães apresentando sintomas depressivos. Entretanto, não foi encontrada associação entre depressão e suporte social ${ }^{23}$. Já Cruz et al. ${ }^{24}$ identificaram, num estudo de corte transversal, com 70 puérperas atendidas nas unidades do Programa de Saúde da Família, índices de depressão materna de 37,1\% no grupo estudado. Além disso, foi constatado que a presença de suporte social do marido estava associada a menor incidência de sintomas depressivos. Finalmente, Moraes et al. ${ }^{25}$ citam a prevalência de $19,1 \%$ numa amostra de 410 mães, associada à renda familiar, sexo da criança e pensamento de interromper a gestação.

No presente estudo, os valores encontrados de $35,7 \%$ de mães com sintomatologia depressiva são expressivos, mesmo se a análise for limitada para as classificações moderada e grave, quando, então, o índice equivale a $17 \%$ da amostra. Além disso, identificou-se que o suporte social foi um fator importante na manifestação depressiva, sendo que mães separadas ou sem companheiros e provenientes de famílias nãonucleares apresentaram maior freqüência de sintomas depressivos.

A falta de suporte e de apoio por parte do companheiro é característica freqüente encontrada em diversos estudos ${ }^{22}$ e em amostras provenientes de diferentes regiões, como é o caso das investigações realizadas na Alemanha ${ }^{26} \mathrm{e}$ na África do Sul${ }^{2}$. Em ambos os casos, a falta de suporte estava associada a maior freqüência de depressão materna, característica que confirma a tendência da literatura na área em identificar relações familiares conflitivas e falta de apoio familiar e do companheiro como fatores contextuais associados aos casos de depressão materna ${ }^{2}$.

O tempo de amamentação exclusiva e total também diferiu aos 6 meses e aos 12 meses nos grupos de mães com sintomas de depressão. Em ambas as situações, houve menor freqüência de aleitamento materno nos grupos de mães com depressão. Uma investigação com 1.745 mães na Austrália também demonstrou que a depressão estava associada ao desmame precoce. E quanto mais cedo ocorria o episódio depressivo, menor era o tempo de amamentação, destacando o impacto negativo da depressão sobre o engajamento materno em práticas de aleitamento ${ }^{27}$.

Este estudo não confirmou a hipótese de que a depressão materna afeta o desenvolvimento da criança. Alguns fatores podem estar relacionados a esse achado, como o tamanho da amostra, a idade da criança e o instrumento utilizado. Cicchetti et al. ${ }^{28}$ também não identificaram diferenças no desenvolvimento cognitivo (Teste Bailey) em um grupo de 97 crianças, aos 18 meses de idade, cujas mães foram identificadas como deprimidas, e em um grupo-controle de 61 crianças com mães sem história atual ou pregressa de transtorno mental. Entretanto, posteriormente, aos 3 anos de idade, o grupo de crianças com mães deprimidas que não havia sido submetido à psicoterapia apresentou escores menores de inteligência no Teste Wechsler de Inteligência. Outro foco importante a ser destacado nesse estudo foi a menor manifestação de emoções em relação à criança observada nas mães com sintomas de depressão. As mães deprimidas referem falta de apoio, isolamento, cansaço e problemas físicos de saúde como fatores que contribuem para o estado depressivo ${ }^{29}$, situações que podem justificar o menor interesse e disposição nas manifestações de carinho em relação à criança.

Em suma, o presente trabalho permitiu concluir que é necessária maior atenção à saúde mental das mulheres, considerando a alta prevalência de sintomas depressivos no grupo estudado e a forte associação com o menor tempo de aleitamento materno, situação que implica em maior risco à saúde da criança, especialmente nos estratos socioeconômicos menos privilegiados. A estrutura familiar foi importante no contexto dos sintomas depressivos, sugerindo que esse aspecto deve ser considerado pelos profissionais responsáveis pela saúde materno-infantil.

Assim, é interessante o desenvolvimento de protocolos com mães e gestantes abordando a questão da depressão sob uma perspectiva multidimensional, considerando-se os diferentes níveis do problema, desde a situação individual materna às questões da relação com o pai e familiares. Além disso, enfatiza-se o fato de que a maioria dos casos não é detectada e permanece sem tratamento, constando-se que a depressão materna em grupos vulneráveis é um problema de saúde grave, que necessita ser identificado precocemente. Somente dessa forma serão possíveis a deteç̧ão precoce e o desenvolvimento de ações que certamente terão impacto na qualidade do desenvolvimento infantil.

\section{Referências}

1. Motta MG, Lucion AB, Manfro GG. Efeitos da depressão materna no desenvolvimento neurobiológico e psicológico da criança. Rev Psiquiatr RS. 2005;27(2):165-76.

2. Cooper PJ, Tomlinson M, Swartz L, Woolgar M, Murray L, Molteno C. Post-partum depression and the mother-infant relationship in a South African peri-urban settlement. Br J Psychiatry. 1999;175:5548.

3. Cooper PJ, Murray L. Postnatal depression. BMJ. 1998;316(7148):1884-6.

4. Murray L, Hipwell A, Hooper R, Stein A, Cooper P. The cognitive development of 5-year-old children of postnatally depressed mothers. J Child Psychol Psychiatry. 1996;37(8):927-35.

5. Field T. Infant of depressed mothers. Inf Behav Dev. 1995;18:1-13.

6. Luoma I, Kaukonen P, Mantymaa M, Puura K, Tamminen T, Salmelin R. A longitudinal study of maternal depressive symptoms, negative expectations and perceptions of child problems. Child Psychiatry Hum Dev. 2004;35(1):37-53. 
7. Schwengber DDS, Piccinini CA. O impacto da depressão pós-parto para a interação mãe-bebê. Estud Psicol. 2003;8(3):403-11.

8. Donovan WL, Leavitt LA, Walsh RO. Conflict and depression predict maternal sensitivity to infant cries. Inf Behav Dev. 1998;21(3):50517.

9. Leiferman J. The effect of maternal depressive symptomatology on maternal behaviors associated with child health. Health Educ Behav. 2002;29(5):596-607.

10. Field T, Hernandez-Reif M, Feijo L. Breastfeeding in depressed mother-infant dyads. Early Child Dev Care. 2002;172(6):539-45.

11. De Rivieres-Pigeon C, Saurel-Cubizolles MJ, Lelong N. Considering a simple strategy for detection of women at risk of psychological distress after childbirth. Birth. 2004;31(1):34-42.

12. Seimyr L, Edhborg M, Lundh W, Sjögren B. In the shadow of maternal depressed mood: experiences of parenthood during the first year after childbirth. J Psychosom Obstet Gynaecol. 2004;25(1):2334.

13. World Health Organization. Postnatal depression: a review. Copenhagen: WHO Regional Office for Europe; 1991.

14. Brasil, Ministério da Saúde. Guia alimentar para crianças menores de 2 anos. Brasília: Ministério da Saúde; 2002.

15. Vitolo MR, Bortolini GA, Feldens CA, Drachler ML. Impactos da implementação dos dez passos da alimentação saudável para crianças: ensaio de campo randomizado. Cad Saude Publica. 2005;21(5):144857.

16. Beck AT, Steer RA. Beck depression inventory manual. San Antonio: The Psychological Corporation; 1987.

17. Frankenburg WK, Thornton SM. Denver developmental activities: a preliminary report. J Dev Behav Pediatr. 1989;10(1):22-6.

18. Drachler ML. Estudo do desenvolvimento das crianças de Porto Alegre: uma contribuição à abordagem epidemiológica. [dissertação]. Porto Alegre: Universidade Federal do Rio Grande do Sul; 1992.
19. Gottfried AW. Home environmental and early cognitive development. Califórnia: Academic Press; 1984.

20. Mundfrom D, Bradley HR. A factor analytic study of the infanttoddler and early childhood versions of the home inventory. Educ Psychol Meas. 1993;53(2):479-89.

21. Patel V, Rodrigues M, DeSouza N. Gender, poverty, and postnatal depression: a study of mothers in Goa, India. Am J Psychiatr. 2002;159(1):3-7.

22. Rahman A, Iqbal Z, Harrington R. Life events, social support and depression in childbirth: perspectives from a rural community in the developing world. Psychol Med. 2003;33(7):1161-7.

23. Coutinho DS, Baptista MN, Morais PR. Depressão pós-parto: prevalência e correlação com o suporte social. Infanto Rev Neuropsiquiatr Infanc Adolesc. 2002;10(2):63-71.

24. Cruz EBS, Simões, GL, Faisal-Cury A. Rastreamento da depressão pós-parto em mulheres atendidas pelo Programa de Saúde da Família. Rev Bras Ginecol Obstet. 2005;27(4):181-8.

25. Moraes IGS, Pinheiro RT, Silva RA, Horta BL, Sousa PLR, Faria AD. Prevalência da depressão pós-parto e fatores associados. Rev Saude Publica. 2006;40:65-70.

26. Herwig JE, Wirtz M, Bengel J. Depression, partnership, social support, and parenting: interaction of maternal factors with behavioral problems of the child. J Affect Disord. 2004;80(2-3):199-208.

27. Henderson JJ, Evans SF, Straton JA, Priest SR, Hagan R. Impact of postnatal depression on breastfeeding duration. Birth. 2003;30(3):175-80.

28. Cicchetti D, Rogosch FA, Toth SL. The efficacy of toddler-parent psychotherapy for fostering cognitive development in offspring of depressed mothers. J Abnorm Child Psychol. 2002;28(2):135-48.

29. Lumley J, Small R, Brown S, Watson L, Gunn J, Mitchell C, et al. PRISM (Program of Resources, Information and Support for Mothers) Protocol for a community-randomised trial. BMC Public Health. 2006;6:36. 